\title{
STM Study on the (100) Surface of the Ag-In-Yb 1/1 Cubic Approximant
}

\author{
C. Cui ${ }^{a, b, *}$, H. Sharma ${ }^{c}$, P.J. Nugent ${ }^{c}$, M. Shimoda ${ }^{d}$ And An Pang Tsai ${ }^{b, d}$ \\ ${ }^{a}$ Department of Physics, Zhejiang Sci-Tech University, Hangzhou 310018, China \\ ${ }^{b}$ Institute of Multidisciplinary Research for Advanced Materials, Tohoku University, Sendai 980-8577, Japan \\ ${ }^{c}$ Surface Science Research Centre and Department of Physics, The University of Liverpool, Liverpool L69 3BX, UK \\ ${ }^{d}$ National Institute for Materials Science, 1-2-1 Sengen, Tsukuba, Ibaraki 305-0047, Japan
}

\begin{abstract}
Scanning tunneling microscopy studies have revealed a periodic step-terrace structure in the (100) surface of the Ag-In-Yb $1 / 1$ cubic approximant. The step height between the large terraces is about $0.73 \mathrm{~nm}$, approximately one-half of the lattice constant, which is consistent with the bcc-like crystalline structure of this crystal. Two small terraces are observed between the adjacent large terraces. High-resolution STM images of the large terrace exhibit a square lattice of protrusions with a lattice constant of about $1.55 \mathrm{~nm}$, which is consistent with the bulk lattice constant. The scanning tunneling microscopy results suggest that the large terraces are associated with the planes that intersect the centers of rhombic triacontrahedral clusters (the building unit of the crystal) and show the highest atomic density along the [100] axis. It is highly likely that the small terraces are related to moderate atomic density planes and are less stable than the large terraces.
\end{abstract}

DOI: $10.12693 /$ APhysPolA.126.577

PACS: 61.44.Br, 68.35.B-, 68.37.Ef

\section{Introduction}

Quasicrystal surfaces have attracted considerable attention due to their novel quasicrystalline surface structure and related physical and chemical properties [1, 2]. However, the surface structure of quasicrystals has so far resisted investigation due to their aperiodic bulk structure and the lack of a precise structure model. Approximant crystals are intermetallic compounds whose compositions and structures are similar to those of their parent quasicrystals. Since approximants possess periodicity, they play a crucial role in understanding the complex surface structure of quasicrystals [1-4].

The icosahedral $\mathrm{Cd}-\mathrm{Yb}(i-\mathrm{Cd}-\mathrm{Yb})$ quasicrystal is a novel binary quasicrystal with high chemical order and thermodynamic stability [5-7]. An extensive family of quasicrystals isostructural to $i-\mathrm{Cd}-\mathrm{Yb}$ and related approximants has been also discovered. The $i-\mathrm{Ag}-\mathrm{In}-\mathrm{Yb}$ quasicrystal was obtained by replacing $\mathrm{Cd}$ with an equivalent amount of $\mathrm{Ag}$ and In [8]. Two kinds of cubic approximant crystals (the $1 / 1 \mathrm{Ag}_{40} \mathrm{In}_{46} \mathrm{Yb}_{14}$ approximant and $2 / 1 \mathrm{Ag}_{41} \mathrm{In}_{44} \mathrm{Yb}_{15}$ approximant) were also obtained $[9,10]$. These $\mathrm{Ag}-\mathrm{In}-\mathrm{Yb}$ quasicrystals and approximants are suitable for surface studies that require ultrahigh vacuum environments, since they contain no high-vapor-pressure elements such as cadmium.

We recently studied the structure of the fivefold, threefold and twofold surfaces of $i$ - $\mathrm{Ag}-\mathrm{In}-\mathrm{Yb}$ using scanning tunneling microscopy (STM) and low-energy electron diffraction (LEED), and discovered that these high-

*corresponding author; e-mail:

cancui@zstu.edu.cn; msecuican@hotmail.com -symmetry surfaces commonly exhibit step-terrace structures with large flat terraces separated by different step-heights [11-14]. High-resolution STM images also show that the quasicrystal surfaces are terminated at planes that intersect the centers of rhombic triacontrahedral (RTH) clusters, the main building blocks of this quasicrystal. This result suggests that the structure of the RTH cluster is related to the formation of stable surfaces. However, it is difficult to make a rational analysis of the stability of a particular plane in quasicrystals, because, unlike in periodic systems, no two planes are identical. Furthermore, the acute rhombohedron (AR) [7], which is another building block of this quasicrystal that fills the spaces among RTH clusters, generates more complexity in analyses.

In this work, we present the first report on the surface of the $1 / 1$ cubic approximant crystal in this quasicrystal family. The $1 / 1$ approximant phase is regarded as a bcc-packing of the RTH clusters with a lattice constant $a=1.54 \mathrm{~nm}$ [7]. The RTH clusters are connected with each other by the $b$-linkage along the [100] direction and the $c$-linkage along the [111] direction [7]. Since RTH clusters are closely packed, there are no large spaces between them, and therefore no AR units are included. Thus, the $1 / 1$ approximant crystal can be regarded as the simplest system built from the RTH clusters and thus is helpful for understanding the surface structure of quasicrystals.

\section{Experimental}

A single-grain $\mathrm{Ag}-\mathrm{In}-\mathrm{Yb} 1 / 1$ cubic approximant crystal was grown using the self-flux method [15]. Figure 1a shows two single-grain approximant crystals. The Laue diffraction pattern in Fig. 1b confirms that the square- 
-shaped facets in the crystal are the (100) planes. Samples were prepared by mechanically polishing a (100) facet with successively finer grades of diamond suspension down to $0.25 \mu \mathrm{m}$. The surfaces were next cleaned in an ultrahigh vacuum (UHV) chamber with a base pressure of $2 \times 10^{-10}$ mbar by repeated cycles of $\mathrm{Ar}^{+}$sputtering at $3 \mathrm{keV}$ for $30 \mathrm{~min}$ and annealing at $713 \mathrm{~K}$ for $2 \mathrm{~h}$. Typically, three or four cycles of sputtering and annealing were sufficient. The surface cleanliness was confirmed by measuring the core level X-ray photoelectron spectra (XPS) of $\mathrm{Yb}$, the element that, of the constituents, is the most sensitive to oxidation. STM measurements were performed on a clean surface using an Omicron STM operating at room temperature.
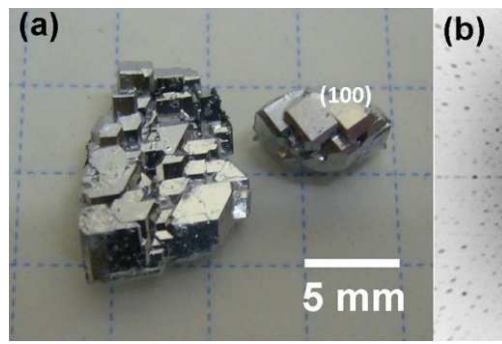

Fig. 1. (a) Ag-In-Yb $1 / 1$ approximant crystals. (b) Back Laue X-ray diffraction pattern of the (100) plane.

\section{Experimental results and discussion}

\subsection{Step-terrace structure}

The clean (100) surface exhibits a clear step-terrace structure. Figure 2 shows typical STM images and related line profiles across the terraces. In a large-scale STM image (Fig. 2a), large terraces are observed that have a constant step height. The details of the step-terrace structure are shown in a magnified STM image in

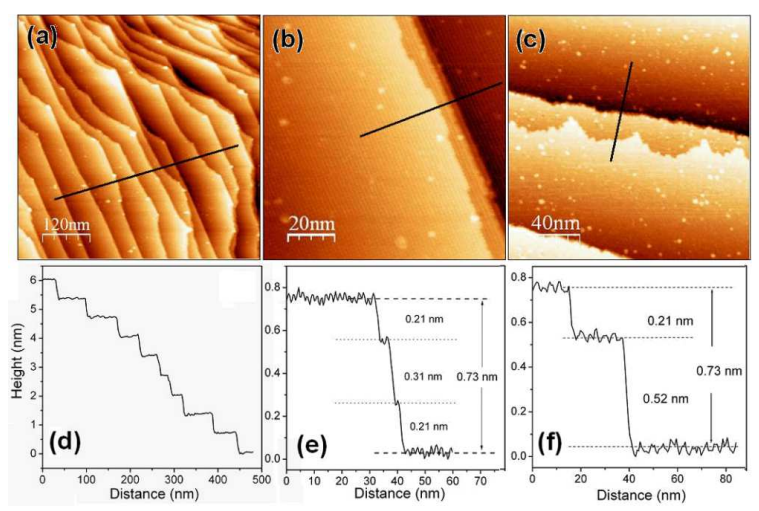

Fig. 2. STM images of the (100) plane of $\mathrm{Ag}-\mathrm{In}-\mathrm{Yb}$ 1/1 approximant crystal: (a) $600 \mathrm{~nm} \times 600 \mathrm{~nm}$ (b) $100 \mathrm{~nm} \times 100 \mathrm{~nm},($ c) $200 \mathrm{~nm} \times 200 \mathrm{~nm}$. (d)-(f) Line profiles along the lines in images (a)-(c), respectively.
Fig. 2b. It appears that the large steps (around $0.73 \mathrm{~nm}$ ) between adjacent large terraces in Fig. 2a are in turn composed of three small steps of $0.21,0.31$, and $0.21 \mathrm{~nm}$ (Fig. 2e). The terrace that appears at $0.21 \mathrm{~nm}$ below the large terrace is small and the area ratio of this small terrace to the large terrace is about 0.08 . The other small terrace, appearing at $0.52 \mathrm{~nm}$ below the large terrace, is much narrower and even absent in some STM images (see Fig. 2c and $\mathrm{f}$ ). The area ratio to the large terrace is almost negligible. The small values of the area ratio suggest these small terraces to be thermodynamically less stable than the large terraces.

\subsection{High-resolution STM images}

Figure 3a shows a high-resolution STM image of the large terrace. Unlike for the $i-\mathrm{Ag}-\mathrm{In}-\mathrm{Yb}$ quasicrystal surfaces, atomic resolution images were not obtained on the approximant surface for any of the tunneling conditions adopted. Instead, a square lattice of protrusions was observed in the large terraces. The distance between the protrusions is around $1.55 \mathrm{~nm}$, which is consistent with the lattice constant of the approximant. The inset in Fig. 3a shows a Fourier transform (FT) of the STM image, which confirms the long-range order of the square lattice. Figure $3 \mathrm{~b}$ shows an STM image in which the bias voltage from the tip to the sample was changed from positive to negative during scanning. Unlike the quasicrystalline surface of $i$ - $\mathrm{Ag}-\mathrm{In}-\mathrm{Yb}[12,13]$, no significant difference can be seen between the positive and negative bias images, except for a small difference in image contrast. It should be noted that both the unattainability of atomic resolution and that of bias dependence are also true for the $\mathrm{Ag}-\mathrm{In}-\mathrm{Gd}$ and the $\mathrm{Ag}-\mathrm{In}-\mathrm{Tb}$ crystals [16]. These are approximant crystals of the Ag-In-Yb family, generated by replacing $\mathrm{Yb}$ with $\mathrm{Gd}$ and $\mathrm{Tb}$, respectively. These results suggest that the electrical states of the approximant are different from those of the quasicrystal at the surface.

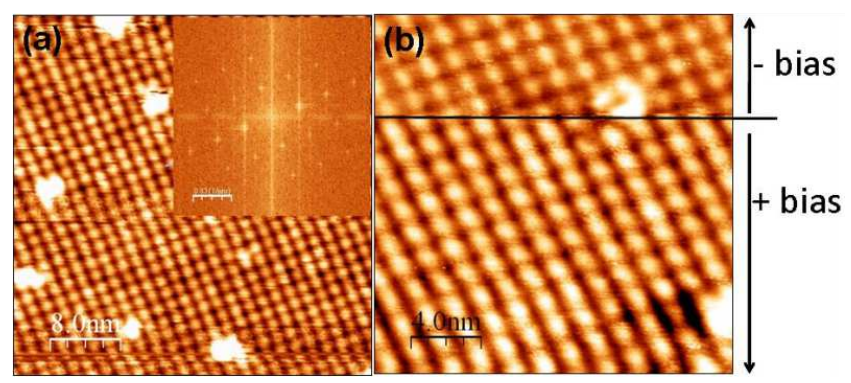

Fig. 3. (a) High-resolution STM image of the (100) plane $(-0.66 \mathrm{~V}, 40 \mathrm{~nm} \times 40 \mathrm{~nm})$ and FT of the image. (b) STM image taken at different bias (from $+0.95 \mathrm{~V}$ to $-0.95 \mathrm{~V}, 20 \mathrm{~nm} \times 20 \mathrm{~nm}$ ).

\subsection{Terraces in the structure model}

Figure 4 shows the atomic density distribution along the [100] axis of the $\mathrm{Ag}-\mathrm{In}-\mathrm{Yb} 1 / 1$ approximant structure model. The highest density appears at the planes that 
intersect the cluster centers (C.C.-intersecting planes). The distance between two C.C.-intersecting planes is $0.77 \mathrm{~nm}$, which is in good agreement with the observed large step height of $0.73 \mathrm{~nm}$. Since termination at the high-density planes is the general pattern in crystals, it is reasonable to conclude that the large terraces correspond to the C.C.-intersecting planes. This would indicate that the same selection rule of the stable surface as for the quasicrystal surface applies to the approximant surface [12-14]. It is also likely that the protrusions observed in the large terraces are related to RTH clusters.

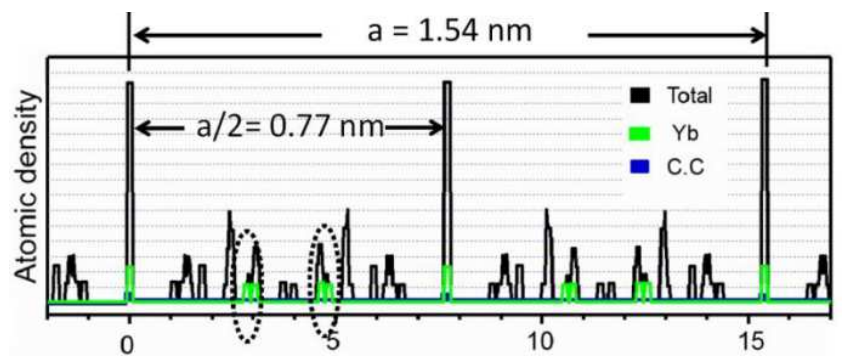

Fig. 4. The atomic density of $\mathrm{Ag}-\mathrm{In}-\mathrm{Yb} 1 / 1$ approximant crystal along the $[100]$ axis. The highest density planes are the C.C.-intersecting planes.

Although the same selection rule controls the termination, the surface of the approximant can be chemically different from that of the quasicrystal surface. This is because the spatial arrangement of each cluster is different from the others: bcc-packing for the approximant and quasiperiodic for the quasicrystal. The C.C.-intersecting planes also intersect the ARs in the quasicrystal, while no ARs are present in the approximant. Thus, different electrical states are expected at the surface between the approximant and the quasicrystal, which causes the unattainability of atomic resolution and of bias dependence for the approximant. Further discussions will require a full evaluation of the stable surface on the basis of first-principles calculations: these are in progress.

The density distribution shows that a large number of atoms cluster around $0.28 \mathrm{~nm}$ below or above the C.C.-intersecting planes and form moderate-density layers (highlighted with dashed ovals in Fig. 4). Taking the distance from the C.C.-intersecting planes into account, it is clear that these moderate-density layers are responsible for the formation of the small terraces. It should be pointed out that the density of $\mathrm{Yb}$ is high both at the C.C.-intersecting planes and the moderate-density layers (Fig. 4). This reminds us of the finding that, in $i$ - Ag-In-Yb quasicrystal, surface termination occurs at the C.C.-intersecting planes where the density of $\mathrm{Yb}$ is also high [12]. It thus appears likely that the $\mathrm{Yb}$ atom or the $\mathrm{Yb}$ shell (the third shell of the RTH cluster) plays an important role in the formation of a stable surface.

\subsection{Impurity phase}

In addition to the inherent surface structure of the (100) plane, step-terrace structures of an impurity phase were also observed (Fig. 5). These exhibit a unique step height of $0.25 \mathrm{~nm}$, which has no relationship with the approximant structure. No fine structure could be observed on the terraces. Dislocations are frequently observed in the impurity phase. The impurity phase might be related to $\mathrm{Ag}$-In alloys, which usually accompany the $\mathrm{Ag}-\mathrm{In}-\mathrm{Yb}$ approximant phase due to the lack of $\mathrm{Yb}$, which was preferentially removed during the sputtering process of surface preparation [10].

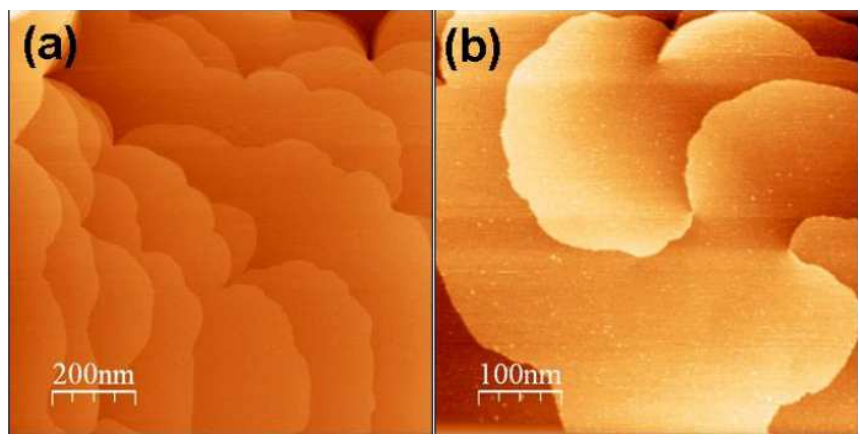

Fig. 5. STM images of the impurity phase in the approximant crystal surface. (a) $1000 \mathrm{~nm} \times 1000 \mathrm{~nm}$ and (b) $500 \mathrm{~nm} \times 500 \mathrm{~nm}$.

\section{Conclusion}

STM images reveal a periodic step-terrace structure in the (100) plane of the $\mathrm{Ag}-\mathrm{In}-\mathrm{Yb} \mathrm{1/1}$ approximant crystal. The step height between the large terraces is close to one-half of the lattice constant which is consistent with the bcc-like structure of the approximant. High-resolution STM images of the large terraces show a square lattice of protrusions that are also consistent with the structure of the approximant. A comparison of STM images with the bulk structure suggests that the large terraces correspond to high atomic density planes intersecting the centers of RTH clusters and that the protrusions are related to the RTH clusters. Two small terraces are observed between the adjacent large terraces. It is reasonable to conclude that these terraces are associated with the moderate-density layers and are thus likely to be less stable than the large terraces. The unattainability of the atomic resolution and of the bias dependence suggests that the electrical states of the approximant are different from those of the quasicrystal at the surface.

\section{Acknowledgments}

This work was supported by the Global COE Program "Materials Integration (International Center of Education and Research), Tohoku University," MEXT, Japan. C.C. acknowledges the support of the National Natural Science Foundation of China (No. 11074220), the Natural Science Foundation of Zhejiang Province (No. Y4100310), the Qianjiang Talent Program of Zhejiang Province (2013R10060), and SRF for ROCS and SEM. 


\section{References}

[1] H.R. Sharma, M. Shimoda, A.P. Tsai, Adv. Phys. 56, 403 (2006).

[2] P.A. Thiel, Annu. Rev. Phys. Chem. 59, 129 (2008).

[3] V. Fournée, A.R. Ross, T.A. Lograsso, J.W. Anderegg, C. Dong, M. Kramer, I.R. Fisher, P.C. Canfield, P.A. Thiel, Phys. Rev. B 66, 165423 (2002).

[4] H.R. Sharma, M. Shimoda, V. Fournée, A.R. Ross, T.A. Lograsso, A.P. Tsai, Phys. Rev. B 71, 224201 (2005).

[5] A.P. Tsai, J.Q. Guo, E. Abe, H. Takakura, T.J. Sato, Nature 408, 537 (2000).

[6] J.Q. Guo, E. Abe, A.P. Tsai, Phys. Rev. B 62, 14605 (2000).

[7] H. Takakura, C.P. Gomez, A. Yamamoto, M. de Boissieu, A.P. Tsai, Nature Mater. 6, 58 (2007).

[8] J.Q. Guo, A.P. Tsai, Philos. Mag. Lett. 82, 349 (2002).

[9] S. Ohhashi, J. Hasegawa, S. Takeuchi, A.P. Tsai, Philos. Mag. 87, 3089 (2007).
[10] C. Cui, A.P. Tsai, J. Cryst. Growth 312, 131 (2010).

[11] H.R. Sharma, M. Shimoda, S. Ohhashi, A.P. Tsai, Philos. Mag. 87, 2989 (2007)

[12] H.R. Sharma, M. Shimoda, K. Sagisaka, H. Takakura, J.A. Smerdon, P.J. Nugent McGrath, D. Fujita, S. Ohhashi, A.P. Tsai, Phys. Rev. B 80, 121401 (2009).

[13] C. Cui, P.J. Nugent, M. Shimoda, A.P. Tsai, R. McGrath, H.R. Sharma, J. Phys., Condens. Matter 24 445011 (2012).

[14] P.J. Nugent, J.A. Smerdon, R. McGrath, M. Shimoda, C. Cui, A.P. Tsai, H.R. Sharma, Philos. Mag. 91, 2862 (2011).

[15] C. Cui, A.P. Tsai, J. Alloys Comp. 536, 91 (2012).

[16] S.S. Hars, H.R. Sharma, J.A. Smerdon, T.P. Yadav, R. Tamura, M. Shimoda, R. McGrath, Acta Phys. Pol. A 126, 479 (2014). 